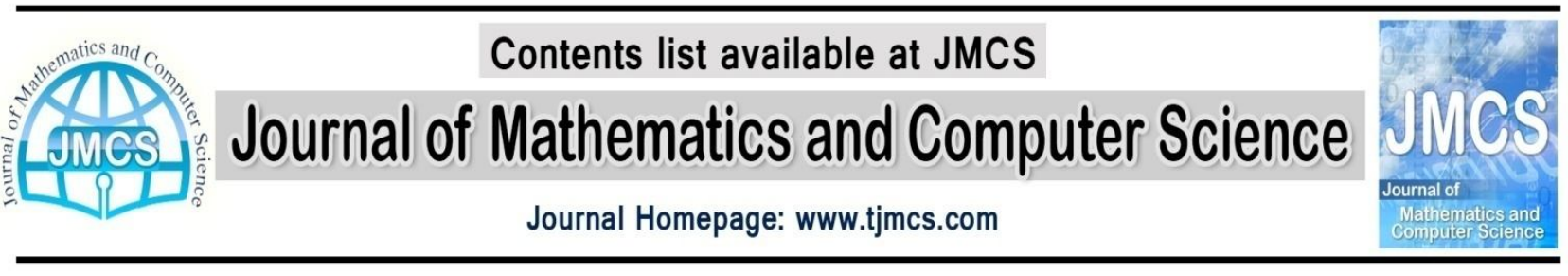

\title{
Application of Fuzzy Logic on Repairing Robotic of Nuclear Power Plants
}

\author{
Jamal Ghobadi Dizaj Yekan \\ Department of Electrical Engineering, Marand Branch, Islamic Azad University, Marand, Iran \\ Jamal_Ghobadi@yahoo.com \\ Amir Torabi \\ Khorasan Institute of Higher Education, Mashhad, Iran \\ Amirtorabi@gmail.com \\ Seyed Kamaleddin Mousavi Mashhadi \\ Iran University of Science and Technology, school of Electrical Engineering, Tehran, Iran \\ Sk_Mousavi@iust.ac.ir
}

Article history:

Received December 2013

Accepted January 2014

Available online January 2014

\begin{abstract}
Today, the usage of robots in the industry has remarkably increased. Among the industries where the hazard exists and the use of robots has a lot of attention, is the nuclear industry. This can cause to prevent a person to have direct contact with nuclear radiation. To control the robot the PID control method is used up to now. This controller has some limitations which make it restricted in order to control complex process. In this paper, instead of PID controllers for process control fuzzy controller is used. To prove the superiority of the fuzzy controller the simulated output is demonstrated. In this paper a fuzzy controller based on the regression model has been studied and Simulation of the relevant section is displayed. Regarding to the use of various compounds of uranium, toxic materials in the nuclear fuel cycle facilities, all necessary safety considerations at the design stage must be considered to prevent accident occurring. Since the nuclear fuel cycle facilities are classified in categories of chemical facilities, Safety Tips is similar to other chemically facilities. One of the ways by which the risk of contact with contaminated sites and structures are reduced, is the use of robots instead of humans in these facilities. Robots are now used in many industrial applications
\end{abstract}

Keywords: Fuzzy Controller, PID Controller, Robots 


\section{Introduction.}

Due to the toxicity of various compounds of uranium materials at nuclear fuel cycle facilities, safety considerations must all be considered in the design phase prevent accidents caused by them. . Since nuclear fuel cycle facilities, chemical facilities are classified as been considered chemical considerations, safety considerations, it is chemically similar to other utilities. Employees working at the facility due to possible leakage of toxic materials in the nuclear fuel cycle facilities, there are always at risk. [13]

Robots are now used in many industrial applications. In some cases, sensitive robots need quick answer a robot that previously used were in some cases able to satisfy this need not in this paper address this problem, the proposed introduction of fuzzy logic Venice PID controller described their advantages of PID fuzzy controller based on perfect simulation explains.

\section{PID controller}

The PID controller is widely used in many industrial uses and in most cases has an acceptable response. This controller has three parts of proportional, integral and derivative. Its block diagram is shown below.

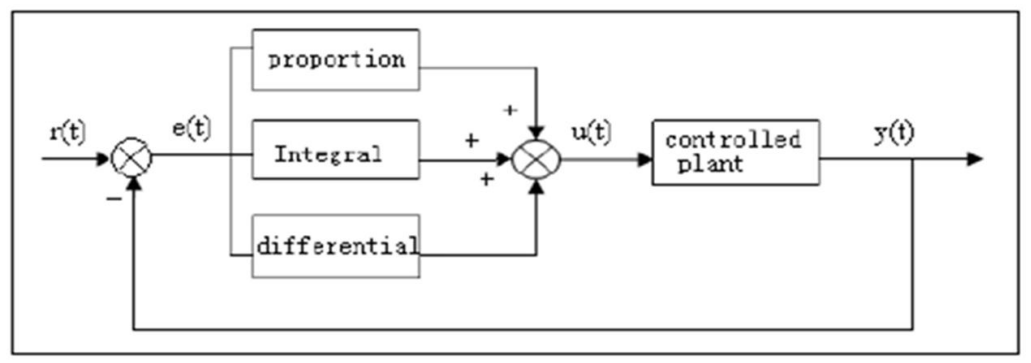

Figure 1.Conventional controller output [2]

From figure 1 controller output $u(t)$ is calculated as follows:

$$
\mathrm{u}(\mathrm{t})=\mathrm{Kpe}(\mathrm{t})+\frac{1}{T_{i}} \int_{0}^{t} e(t) d t+T_{d} \cdot \frac{d e(t)}{d t} \frac{1}{T_{i}} \int_{0}^{t} e(t) d t+T_{d} \cdot \frac{d e(t)}{d t}
$$

The proportional part is used to improve the error signal.

The Integral part is used to reduce the steady state error.

The derivative that could reduce the transient error.

As we know the error of PID controller is calculated as follows:

Where e $(t)$ equals the error, $r(t)$ is the optimal value and $y(t)$ is the output.

\section{Fuzzy controller}

Fuzzy controller designation is based on work experience. In many cases in which the problem is not solved by traditional methods fuzzy technology is used. Because the fuzzy technology does not have complex mathematical relations for fuzzy control of nonlinear systems and systems with large disturbances, it has good performance. [2] 


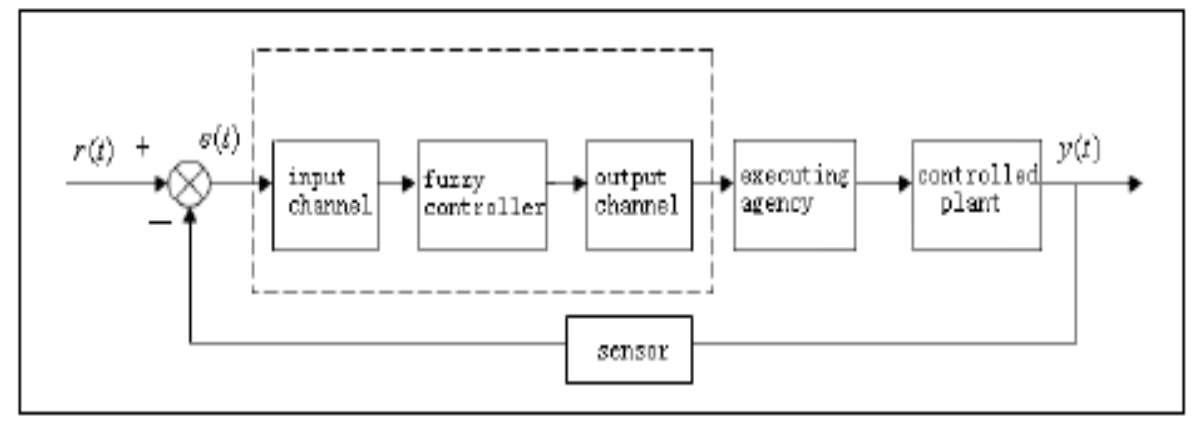

Figure 2: The basic structure of fuzzy controller [11]

The main structure of the fuzzy control system is shown in Figure 2. That has input channel, output channel and fuzzy controller and the fuzzy controller is the core of the system. [2]

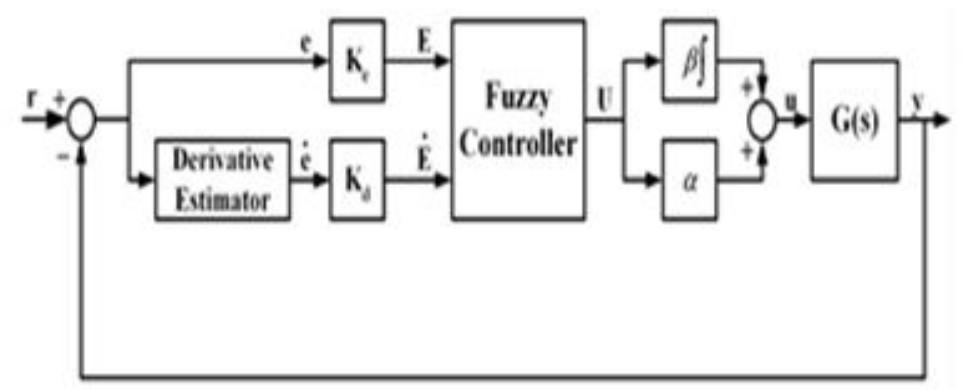

Figure 3: The structure of fuzzy controller with two input changes its error [2]

The structure of the fuzzy controller with two inputs and a rules database is shown in Figure 3.

Design of fuzzy PID controller has two parts. The first part is related to the non-linear part and the Second part is related to adjustment of the coefficient based on the linear part of the system. Rules mentioned in this article are to obtain better the details of properties than conventional PID fuzzy structures. As can be seen in this paper a fuzzy controller has more coefficients compared with PID controller.

Since non-linear controller has non-linear function, it is more difficult than the PID controller to adjust parameters. [6]

\section{Fuzzy robot}

Including discussions about the science of robotics will be considered is robot controlling especially in order to follow the pre designed paths.

In terms of complexity and nonlinear dynamics, and because of static friction, disturbances and sever changing in the parameters robot model, and also the possibility to work in different circumstances and different paths, robot control is very complicated and difficult. Thus, different control methods are presented, each with its own advantages and disadvantages. One of the control methods which had 
tremendous development in the control of complex systems during the last two decades is fuzzy control. Fuzzy controller has two main advantages: these controllers are not sensitive to the model of system and are largely non-dependent to type of relation between input and output of system, and they have very simple structure and are easy to implement. Because the smart robot movements in the race track depend on the programs and track conditions, therefore, very precise formulation of fuzzy rules can be prevented from diversion, as you imagine it is guided by a human.

\section{Fuzzy rule}

As we know that the delay in control systems and the PID control will cause undesirable effect. Types of mechanical systems in this robot is designed to have delay not only in direct but also in the direction of Main feedback. These factors can make the conventional controllers to be unable to control this process. Therefore, in this case (with respect to the cases mentioned above, which would specify the use of fuzzy control) we see the best condition in use of fuzzy controllers.

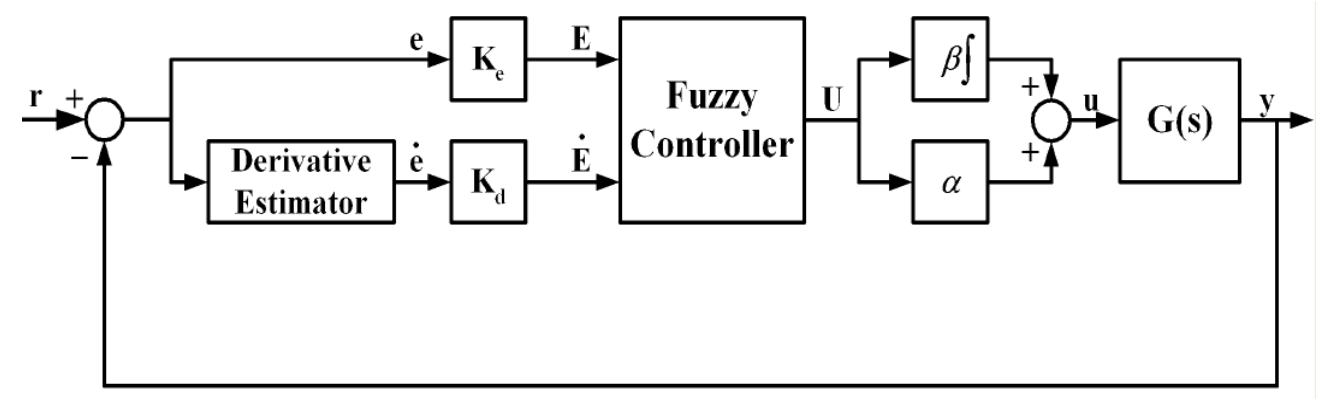

Figure 4: Key to freely choose hybrid or conventional fuzzy controller [10]

As we know, the phase of the required input and output that would be that, as mentioned inputs of e $(t)$ and ệ ( $t$ ) is. Shown in Figure 4 membership functions input in Figure 6 membership functions of output is.

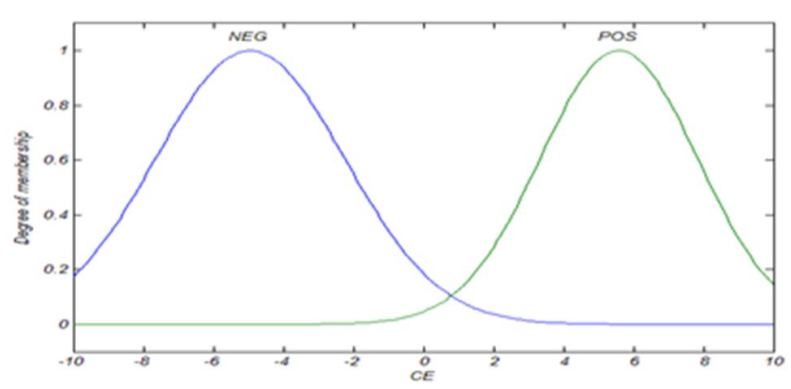

Figure 5: Input membership functions 


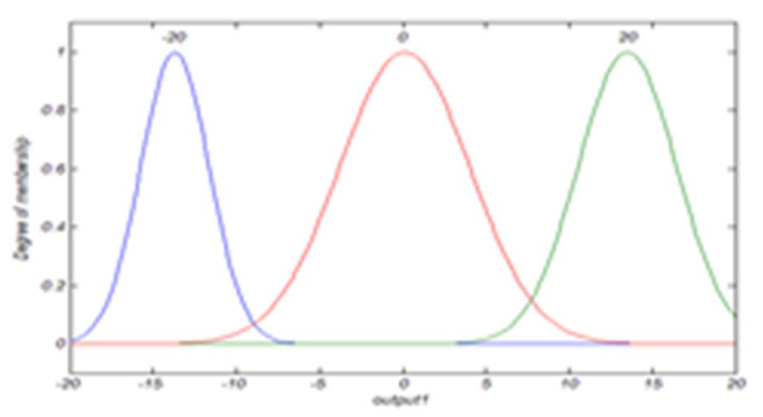

Figure 5: Output membership functions

\section{Regression}

Although the classical linear regression model has many applications but also has problems such as careless behavior, error with assuming linearity, ambiguity in the relationship between input and output variables and we can be created. There for the statistical regression analysis can be problematic. So this situation leads us to the use of fuzzy regression analysis.

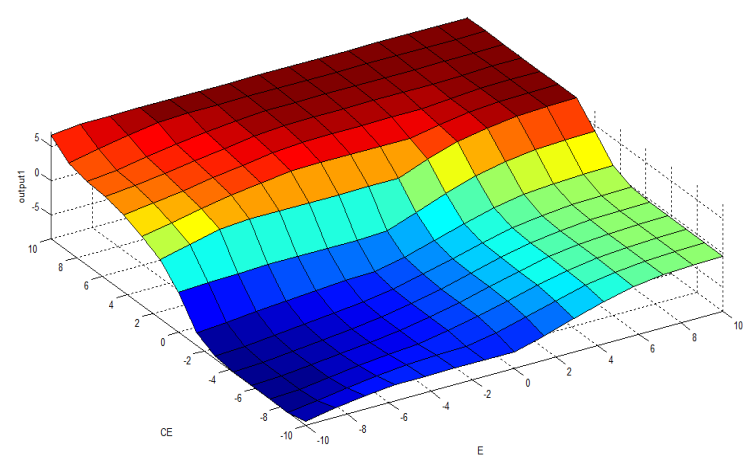

Figure 6: Demonstration surface output

\section{Regression model, the fuzzy controller}

Regression analysis is used to model the relationship between dependent variables and independent variables in order to predicted values of dependent variables on independent variables. In regression analysis the dependent variable $(y)$ is a function of independent variables and degree of participation of each independent variable in the output (dependent variable) is expressed by the coefficients of variables. Regression models are made based on collected data and observations and standard linear regression model in the classic mode is as follows:

$y i=\alpha 0+\alpha 1 x i 1+\ldots+\alpha n x i n+\varepsilon i$

So that the dependent variable (yi), the independent variables (Xij) and the independent model parameters ( $\alpha \mathrm{i})$, are the absolute values and $\mathrm{I}$ is the random of regression models,

$E(\varepsilon i)=0, \quad V(\varepsilon i)=\delta 2$ 


\section{General fuzzy regression model}

So common in regression methods, the difference between observed values and values predicted by the model, are the forecast error and is a random variable. High and low limits of calculated values and probability of being calculated predicted value in between these two limits, expressed confidence in the estimate. Otherwise, the structure of common regression models are based on probability. While in the fuzzy regression, the difference between predicted and observed values of the system is inherently ambiguous. Output value for the specified input range of possible values can be assumed. Therefore, in fuzzy regression, allowing the output value according to the values of input variables are examined.

$\tilde{Y}=f(x, \tilde{A})=\tilde{A}_{1} x_{1}+\cdots+\tilde{A}_{n} x_{n}$

A general model for fuzzy linear regression is as follows Fuzzy regression predicts a range of possible values with a membership function

\section{Fuzzy controller fuzzy regression results}

Based on the regression in Figure 8 above, surface fuzzy controller is shown.

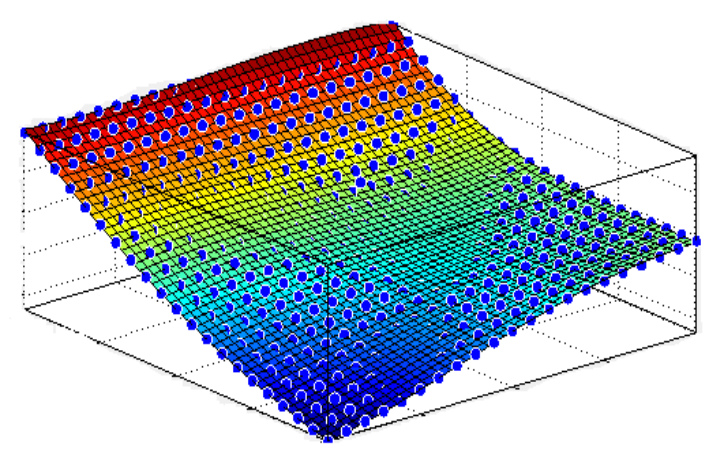

Figure 8: View of fuzzy regression surface

Membership function of output variable regression is obtained as follows

$$
\tilde{Y} \mu(y)=\left\{\begin{array}{cl}
\max \left(\min _{\mathrm{i}}\left(\mu_{\tilde{\mathrm{A}}}(\alpha)\right)\right. & ,\{\mathrm{a} \mid \mathrm{y}=\mathrm{f}(\mathrm{x}, \mathrm{y})\}=\Phi \\
0 & \text { otherwise }
\end{array}\right.
$$

Would be shown as: $\tilde{A}=\{p, c\}$ Ã fuzzy parameters can be vectors

$\mathrm{P}=(\mathrm{P} 1, \mathrm{P} 2, \mathrm{P} 3, \ldots \mathrm{Pn})$

$$
\mathrm{C}=(\mathrm{C} 1, \mathrm{C} 2, \ldots \mathrm{Cn})
$$

By inserting equation (4) in (5) we get:

$\tilde{Y} \mu(y)=\left\{\begin{array}{lr}1-\frac{\left|y-\sum_{i=1}^{n} p_{i} x_{i}\right|}{\sum_{i=1}^{n} c_{i}\left|x_{i}\right|} \quad x_{i} \neq 0 \\ 1 & x_{i}=0, y=0 \\ 0 & x_{i}=0, y \neq 0\end{array}\right.$ 
The controller consists of a linear equation:

$F(X, Y)=P 0+P 1 X+P 2 Y+P 3 X^{\wedge} 2+P 4 X^{*} Y+P 5 Y^{\wedge} 2$

The model coefficients in $\mathrm{X}$ and $\mathrm{Y}$ both have to be two of the simulation.

Errors in the R-square (root mean square) and RMSE (SD) are:

R-square: 0.8848

RMSE: 0.6855

\section{Simulation results:}

PID position controller for the system is designed as follows:

$\mathrm{Gc}(\mathrm{S})=\frac{\mathrm{S}+1}{\mathrm{~S}+0.05}$

In Figure 7, the output of the plant for PID fuzzy controller is shown. Curved yellow red Vmnhny PID controller output for the fuzzy controller for the show.

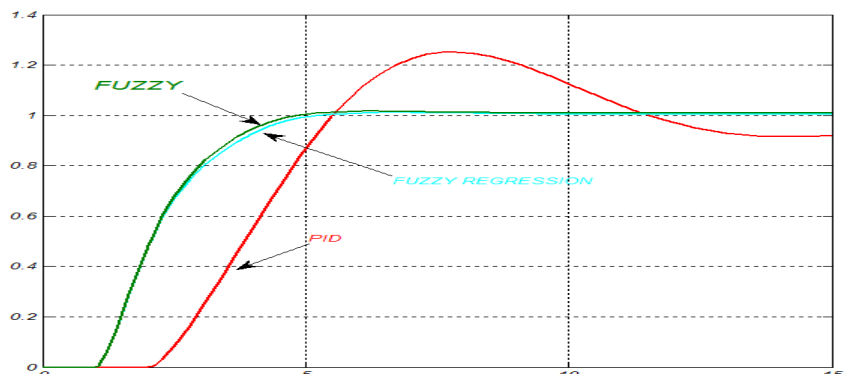

Figure 7: Simulated oscilloscope output Figure 8

At the end of the simulation environment that responds simulink oscilloscope output it can be seen in Figure 7, We show in Figure 8., As we can see in all three categories, each related to one of the fuzzy PID controller is the fuzzy regression. 


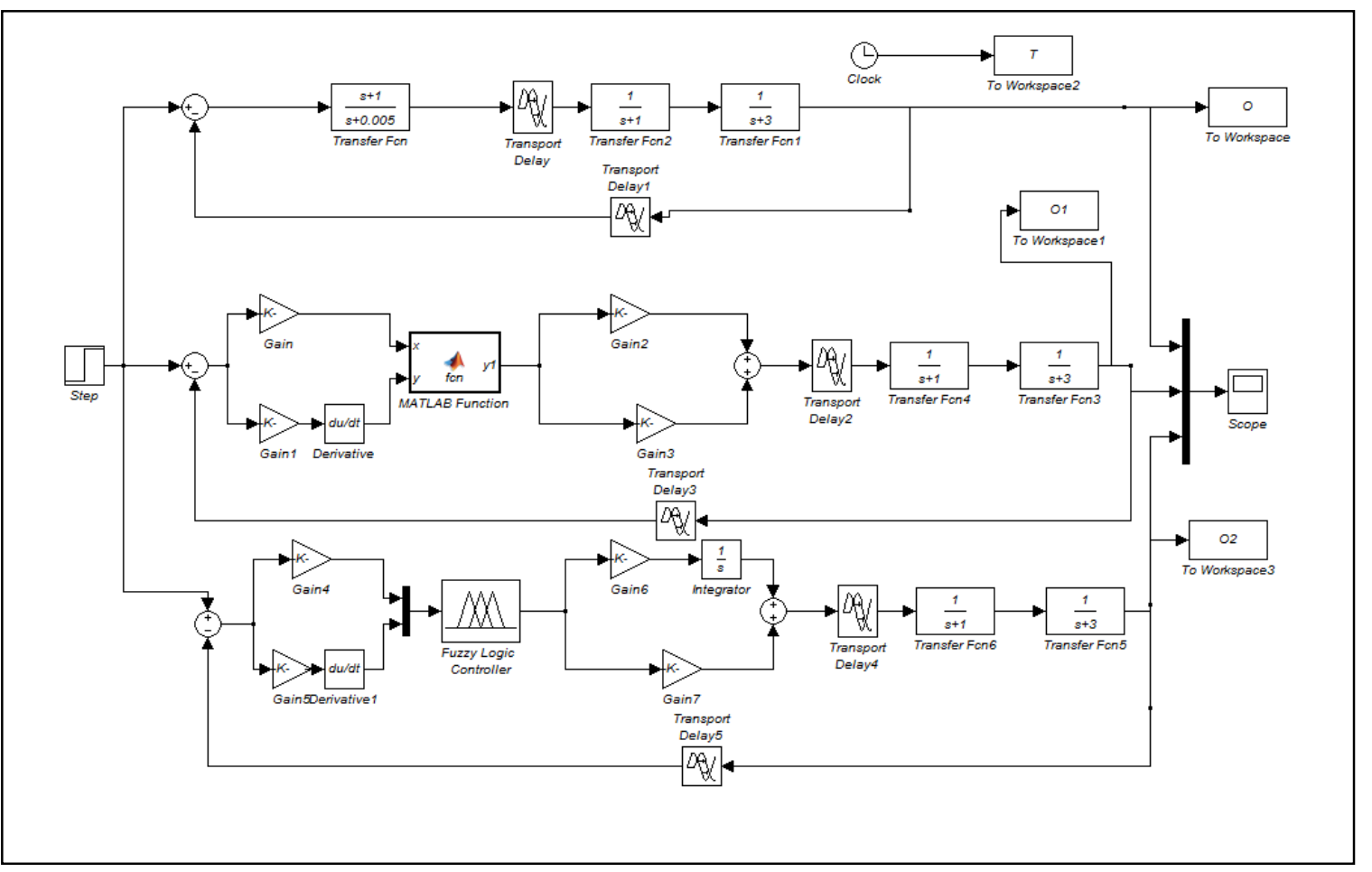

Figure 8: Simulation of fuzzy and PID controller plant and three fuzzy regression

\section{References:}

[1] Isin Erenoglu, Ibrahim Eksin, proceedings, AN INTELLIGENT HYBRID FUZZY PID CONTROLLER, 20th European conference on modeling, Alessandra Orsoni, 2006

[2] Onur KARASAKAL,Turk J Elec Engin, Implementation of a New Self-Tuning Fuzzy PID Controller on PLC, VOL.13, NO.2 2005, TURKEY

[4] Richard Dorf, John Wiley \& Sons, "Original Equipment Manufacturers", Chapter by Kevin J. McDermott. Invited Lecture Series - Beijing Polytechnic University, Bejing, Peoples Republic of China, 1988.

[5] Jan Jantzen, Tuning of Fuzzy PID Controllers, Technical University of Denmark, Department of Automation, Bldg 326, DK-2800, and DENMARK Tech. report no 98-H 871, 30 Sep 1998.

[6] G.K.I. Mann, Hu Bao-Gang, R.G. Gosine, Analysis of Direct Action Fuzzy PID Controller Structures, IEEE TRANSACTIONS ON SYSTEMS, VOL. 29, NO. 3, JUNE 1999.

[7] Y. Yang, W. Yang, M. Wu, Q. Yang, Y. Xue, A New Type of Adaptive Fuzzy PID Controller, IEEE, China 2010.

[8] Hui Wang, Yuanyuan Bao, Fuzzy-PID Dual Mode Fuzzy Control of the Electro-hydraulic Deviation Control System, College of Mechanical Engineering, 2010.

[9] Yanhong Zhang, Zhongqiao Zheng, Jiansheng Zhang, Research on the parameters self-tuning fuzzy PID controller, School of Electronic Information 2009.

[10] Armin Taheri, The Robot Line Finder with Fuzzy Control, The Bachelor, Masters Journal of nuclear safety management and engineering, seventh year No. 26.

[11] B. Kosko, Fuzzy Thinking the New Science of Fuzzy, June 30, 1993.

[12] K. Ogata, Modern Control Systems, Prentice Hall $9^{\text {th }}, 2001$ 